\title{
Multicriteria Parent Selection Using Cognitive Radio for RPL in Smart Grid Network
}

\author{
Adisorn Kheaksong, ${ }^{1}$ Kanabadee Srisomboon, ${ }^{1}$ Akara Prayote, ${ }^{2}$ and Wilaiporn Lee $\mathbb{D}^{1}$ \\ ${ }^{1}$ Communication and Computer Network Research Group (C2NRG), Electrical Engineering, Department of Electrical and \\ Computer Engineering, Faculty of Engineering, King Mongkut's University of Technology North Bangkok, Bangkok, Thailand \\ ${ }^{2}$ Computer Science, Department of Computer and Information Science, Faculty of Applied Science, \\ King Mongkut's University of Technology North Bangkok, Bangkok, Thailand
}

Correspondence should be addressed to Wilaiporn Lee; wilaiporn.l@eng.kmutnb.ac.th

Received 22 August 2017; Revised 8 January 2018; Accepted 23 January 2018; Published 21 February 2018

Academic Editor: Danda B. Rawat

Copyright (C) 2018 Adisorn Kheaksong et al. This is an open access article distributed under the Creative Commons Attribution License, which permits unrestricted use, distribution, and reproduction in any medium, provided the original work is properly cited.

\begin{abstract}
To maintain reliability of advanced metering infrastructure network in smart grid, data sent from a smart meter must reach a data concentrator unit efficiently. Parent selecting mechanism in routing protocol for low-power and lossy (RPL) is a key to maintain the reliability by balancing workload of meters in the network. In this paper, a parent selecting mechanism with three criteria including expected transmission count, residual energy, and expected transmission time is proposed to improve workload balancing and lifetime differences of all meters. A meter selects an immediate parent based on three factors. From simulation results, parents' workload is better balanced and the lifetime of all meters in the network is depleted nearly at the same time. Moreover, a simulation with cognitive radio enabled meters, where data can be transmitted on a licensed channel opportunistically when the channel is not utilized, shows an improvement in the packet delivery ratio.
\end{abstract}

\section{Introduction}

Depending on an increasing of electrical usage, an electrical grid where the grid is connected by high-voltage network to local distribution systems cannot meet the requirement of electrical demand. Smart grid [1-5] is defined as the next generation of electrical grid system which integrates several communication technologies and digital data processing to improve the electric system reliability and efficiency. An advanced metering infrastructure (AMI) $[6-8]$ is the application that allows a two-way communication between inhome smart meters and utilities in order to exchange the information-energy usage, control message, and emergency notification alert-between utility and clients via local access point (data concentrator unit: DCU). Therefore, once a malfunction occurs, it is reported to utility within real-time via AMI network. Then, the utility is able to repair the system remotely or sends a maintenance personnel to repair the system timely. On the other hand, the customer benefits from an immediate electrical usage feedback where they can manage their behavior of electrical utilization in economical purposes.

To support several application of AMI, routing protocol for low-power and lossy (RPL) is standardized by Internet Engineering Task Force (IETF) and is expected to be exploited in practice [9-12]. RPL is defined as a routing protocol based on distance vector, where the data route-directed acyclic graphs (DAG) - is constructed according to routing metrics defined by objective function (OF) [13]. In the RPL protocol, there are two main issues that need to be concerned including the meter (or node) lifetime and the transmission reliability $[14,15]$.

For AMI network in smart grid, low-power smart meters are organized as a rooted tree architecture where DCU is the root. The meter lifetime is the critical issue that affects reliability of smart grid system. To maintain the system reliability, data from meters needs to reach the utility forwarded by DCU. There are two situations where the data from meters cannot be transmitted to DCU. First, the battery of the meter depletes. Once a battery of a meter is depleted, not only the 
meter's data cannot be transmitted to the DCU, but also the data packet of its dependent meters (successors) cannot reach to DCU. Second, the battery of the meter's relay node (or immediate parent) depletes and there is no candidate parent to forward the data from the meter. Consequently, it is up to maintenance to keep monitoring individual meter to avoid battery depletion. Therefore, it has to be mentioned that when a battery of a meter depletes, the data of the meter and its dependent meters cannot reach the DCU. This is one of main issues of AMI networks known as a network partitioning [16].

To facilitate the maintenance job in a battery depletion avoiding, the lifetime of all meters should be nearly the same. Therefore, the workload of meters in the network needs to be balanced. Parent selection mechanism plays an important role in balancing the meters' workload. In a traditional RPL, the immediate parent is selected by a child meter (child node) and determined by the quality of transmission channel only. The quality of transmission channel is based on expected transmission count (ETX). When the quality of transmission channel is best, ETX becomes 1 as the minimum. Once the quality drops, the value of ETX increases. With this mechanism, the meters with the better quality of transmission channel (lower ETX) will be selected as immediate parents and their workload is higher than others so that their battery will deplete fast. While meters with worse quality of transmission channel do not have much workload, their battery will deplete slower. Once the meters' workload are not balanced, the partitioned network will occur when early depletion of high workload parent occurs.

To balance the meters' workload, the workload needs to be equally split by minimizing an influence of channel quality in a parent selection. Early parent selecting functions focus on either channel quality or energy consumption [17-19]. By selecting the parent on energy constrain only, the meters may suffer from efficient route. Later, an energy-efficient oriented algorithm (EERA) [20] is proposed to balance the workload of parent by determining both channel quality and parent's residual energy. However, the appropriate weights have not been investigated and the detected packet delivery ratio (PDR) has not been studied. Moreover, the expected transmission time (ETT) [21], one of the RPL performance metrics, has not been taken into account yet. It should be noticed that several literatures have not considered the ETT because the communication of RPL is based on only a single protocol-Zigbee. By considering only Zigbee protocol, several transmitting constraints are normally the same, that is, channel bandwidth, maximum bit rate, transmit power, and so on. Therefore, ETT has not been taken into account. Based on an emerging of cognitive radio technology, the available licensed band can be exploited. Therefore, RPL protocol can adopt the CR technology to improve the workload balancing and also the PDR.

CR [22-24] is the technology that allows a dynamic utilizing of the unused communication channels by reconfiguring its operating parameters and functionalities. By allowing an unlicensed user to operate on the unused channel, an issue of spectrum scarcity and data congestion can be resolved. To exploit the CR technology, the devices in the network have to comprise cognitive capabilities where changing surrounding spectrum environment can be evaluated in order to determine the appropriate transmission plans. The first standardized air interface for CR network-IEEE 802.22 $[25,26]$ - is based on an opportunistic utilization on the white spaces within the television bands between 54 and $862 \mathrm{MHz}$, especially within rural areas where usage may be lower.

The main contribution of this paper is to improve the workload balancing and lifetime differences of all meters. Therefore, we propose a new parent selection mechanism, multicriteria parent selection (MPS) algorithm, which balances the meter workload considered from three criteria. The three criteria are based on three main RPL performance factors-ETX, residual energy, and ETT. By analyzing the appropriate parent from multiobjective [27-30], the workload of parent meters can be balanced and the best route is selected. Moreover, with CR enabled meters, MPS shows an improvement in the PDR where data is transmitted on a licensed channel opportunistically when the channel is not utilized. As a result, workload balancing and lifetime differences of all meters as compared to traditional RPL mechanism and energy-efficient oriented algorithm (EERA) are improve. In addition, we also investigate the appropriate distance between meters which achieves an acceptable PDR rate where the rate is as high as 0.9 .

The remainder of this paper is organized as follows. Section 2 gives brief introduction RPL protocol. In Section 3, the issues of RPL for smart grid network are described. In Section 4, the framework of MPS algorithm is described in detail. The simulation results are shown in Section 5. Finally, conclusions are presented in Section 6.

\section{IPv6 Routing Protocol for Low-Power and Lossy (RPL)}

RPL [9] is defined as a routing based distance vector protocol that provides an information distribution over a network topology which is constructed dynamically. Once the nodes in RPL topology operate autonomously, it can minimize some data configurations in the nodes by utilizing trickle timer [31]. To implement with AMI network, nodes in the network are constrained by processing power, memory, and energy. The key aspect of RPL protocol is to connect the nodes in the network using directed acyclic graphs (DAG) without any cycle presented. In a DAG, a rooted tree routing topology is constructed and is called destination-oriented directed acyclic graphs (DODAG).

As depicted in Figure 1, the structure of RPL network can be determined as dynamically constructed rooted tree topology where the lowest node transmits the data to the root node via the upper nodes. There are three types of node including gateway, router, and client. In the topology, root node is determined as a topology gateway. The duty of the rest nodes are determined by the distance from the root and are assigned by the level of rank. The level of rank is assigned to each node in the network related to the nodes' position with respect to the root. If a node is far from the root node, a rank level of the node is high. On the other hand, if a node is placed near to the root node, the level of rank is low. Therefore, the level of rank increases in a downward 


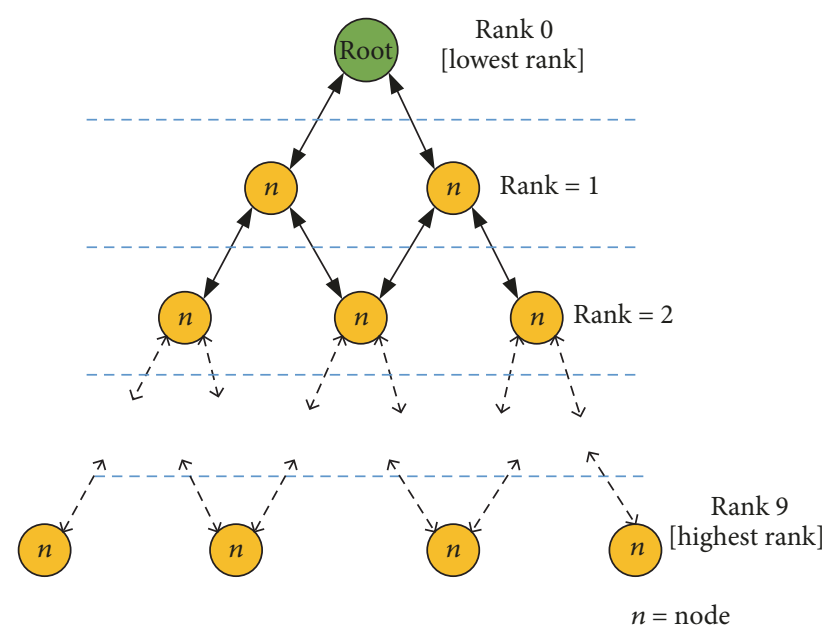

FIGURE 1: Structure of RPL network.

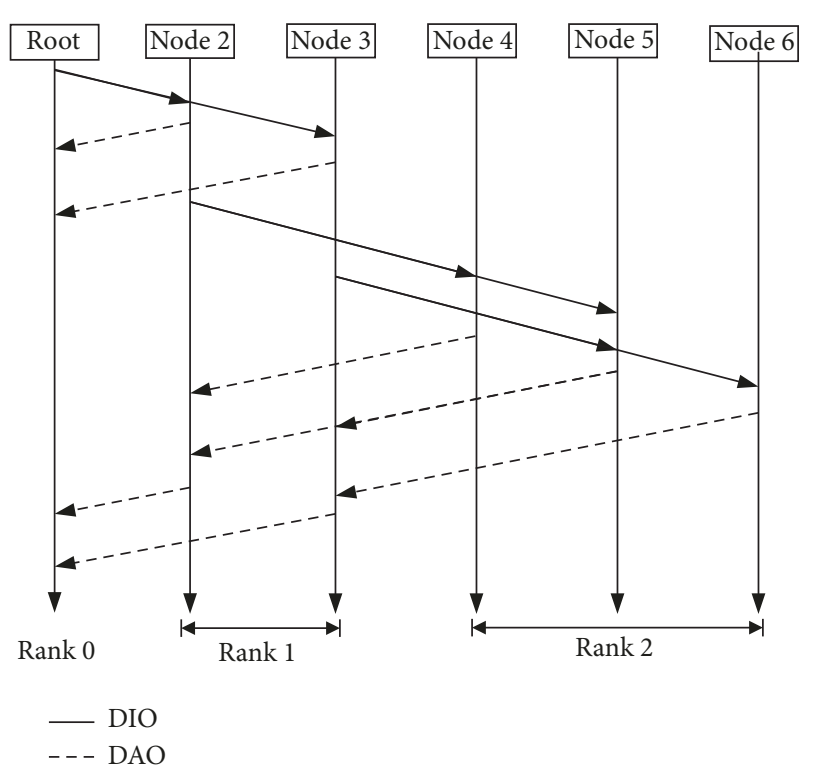

FIGURE 2: Route construction of RPL.

direction with respect to the root node. The node at the lower level of rank services a node at the higher level of rank as a parent. Therefore, the latest level of rank can transmit the data packet toward the root node via several ancestors (lower rank nodes). To prevent a cycle presented, the node in the topology is regulated to associate with only the same or lower level of ranks.

To construct the DAG, three control messages-DAG information option (DIO), destination advertisement object (DAO), and DODAG information solicitation (DIS) - are utilized and the routing metrics are defined by objective function (OF) [13]. First, as depicted in Figure 2, the root node broadcasts a DIO message which contain the information-rank of broadcasting node, OF, and DADAGID-to other nodes in the network. Nodes, which receive DIO message and desire to join the DAG, determine their own rank level using the received DIO message. Moreover,

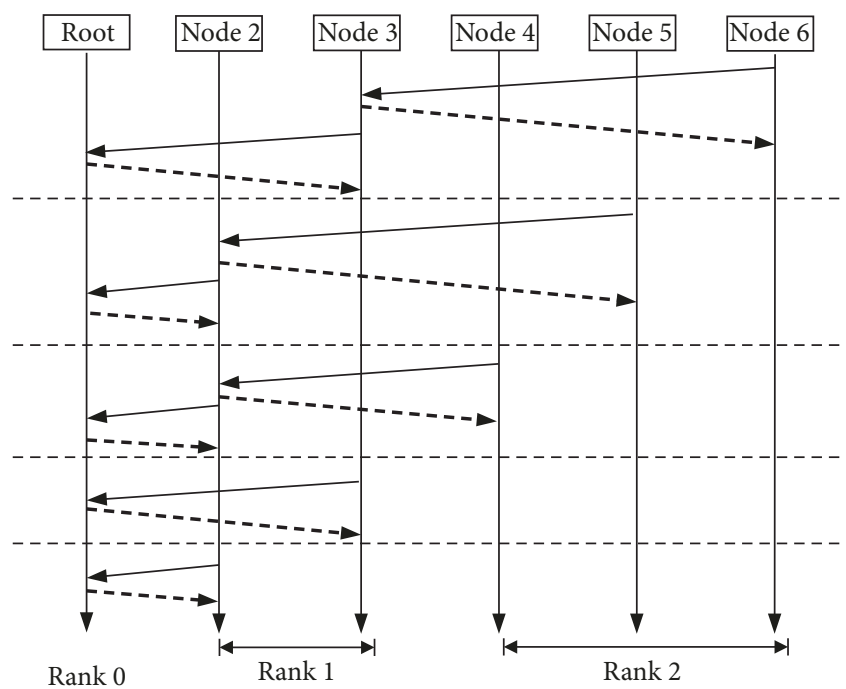

- Data

FIGURE 3: Data transmission period of RPL.

the node which transmitted the DIO message is added to the candidate parent list of receiver node. Once the receiver node receives more than one DIO message, the parent is selected from the candidate parent list constrained by objective metrics in OF. The best transmission channel serviced by the best parent is selected through objective metrics defined by OF. Therefore, a system reliability can be maintained. It should be noted that if the rank of receiver node is not the latest level of rank, it has to update its rank on DIO message and forward it to its neighbors.

To complete the route construction in an outward direction (root to leaf node), DAO message is transmitted in a unicast manner in an upward direction, as shown in Figure 3. In addition, the node will record the information of reverse path. After DAG is completely constructed, the higher level rank nodes are allowed to transmit the data packet in an inward direction-from node to root-via several appropriate ancestors nodes.

In RPL on AMI network, there are three communication schemes-route construction, data transmission, and notification alert transmission. First, DIO and DAO messages are transmitted to construct and maintain the RPL network. Second, based on AMI framework, the energy usage is transmitted from the leaf nodes to the DCU (root node) via their immediate ancestors (a number of parents). In general, the meter has to send the information about energy usage every 15 minutes to the DCU for real-time processing of smart grid technology. However, in this simulation, we set that the meters send the information to the DCU every 5 minutes for evaluating the routing performance. In an RPL protocol, once meters in DODAG have routing entry, the smart meter at the highest rank can send data traffic to the DCU toward immediate parent. Then, the parent will transmit the received data to his parent. It means that the data packet from leaf meter is transmitted to the DCU in 
a hop-by-hop fashion via its ancestors of downward. Third, the notification alert message is transmitted to the DCU which needs to be performed in real-time. In this paper, we focus on route construction and data transmission in order to evaluate the performance of parent selection function. It should be noted that the size of data packet during energy usage transmission interval is larger than route construction; therefore, the size of data packet affects the PDR where the PDR may decrease. Therefore, the PDR of route construction and data transmission has to be investigated individually.

Traditionally, RPL mechanism defines only a single routing metric — the expected transmission count (ETX) - as a constraint for parent selection. ETX is the number of transmissions by a meter and is expected to be received by the destination. Then, the ETX can be expressed as

$$
\operatorname{ETX}=\frac{1}{\left(D_{f} * D_{r}\right)}=\frac{1}{1-p}
$$

where $D_{f}$ is the measured probability that a packet is received by the neighbor, $D_{r}$ is the measured probability that the acknowledgment packet is successfully received, and $p$ is the error rate of data transmission.

In general, the parent is selected by determining ETX of the path where desired value of ETX is as low as 1 that is referred to a high channel quality. To determine the transmission path of each node, an objective function-Minimum Rank with Hysteresis Objective Function (MRHOF) [17] - is considered. To select the smallest path cost, there are two procedures which need to be considered. First, the path with minimum cost is determined. Second, the node selects the minimum cost path if the cost is better than the current path determined by a given threshold. The second procedure is called "hysteresis." Therefore, the route with the smallest path cost is selected. For achieving the objective of RPL, MRHOF can be used with several performance metrics-energy consumption, latency, or ETX-for selecting the best path quality.

As mentioned earlier, by selecting a parent use of a single routing metric cannot achieve the requirements of RPL where the node (meter) lifetime needs to be prolonged while the transmission reliability determined by packet delivery ratio needs to be maintained and stabilized. Therefore, an energyefficient oriented algorithm (EERA) [20] combines these two objectives metrics-ETX and energy-to balance the workload of parent node while selecting the best route. To select the parent, a weight of importance between energy consumption and channel quality is considered. Therefore, a routing score for parent selecting is given by

$R$

$$
\begin{aligned}
= & \alpha \frac{\text { ETX }}{\text { Maximum ETX }} \\
& +(1-\alpha)\left(1-\frac{\text { Remaining Energy }}{\text { Maximum Energy }}\right),
\end{aligned}
$$

Remaining energy $=$ Battery - Energy Consumption where $\alpha$ is the weight of importance between energy and ETX and the term of ETX is an intermediate ETX of a route between candidate parent and node. Once the energy consumption cannot be gathered directly from simulation, it can be calculated by the following equation:

$$
\begin{aligned}
\text { Energy Consumption }= & T_{\mathrm{CPU}} * P_{\mathrm{CPU}}+T_{\text {RadioRX }} \\
& * P_{\text {RadioRX }}+T_{\text {RadioTx }} \\
& * P_{\text {RadioTX }}
\end{aligned}
$$

where $T_{\mathrm{CPU}}$ is the processing time of CPU, $P_{\mathrm{CPU}}$ is the power consumed by CPU, $T_{\text {RadioRX }}$ is the processing time of the receiver, $P_{\text {RadioRX }}$ is the power consumed by receiver, $T_{\text {RadioTX }}$ is the processing time of the transmitter, and $P_{\text {RadioTX }}$ is the power consumed by transmitter.

\section{Problem Statement}

In this section, we describe the issues that affect the performance of RPL protocol of AMI network including meter lifetime and transmission reliability.

3.1. Meter Lifetime. In this subsection, we describe the effect of the nonbalancing of energy. Based on a binary tree structure of AMI network, the root node acts as a DCU responsible for collecting the data from other nodes and also constructing the RPL process. The rest of nodes (or the node in the other ranks) act as an in-home smart meter.

As shown in Figure 4(a), during data transmission process, each node forwards the data to the root node via its immediate parents where the immediate parent is chosen in the route maintenance process. In this case, nodes 4 and 5 choose node 2 as their immediate parent and node 6 chooses node 3 to be its parent. As the passing of time, the battery of node 2 is consumed faster than node 3 . Then, an early depletion of node occurs when node 2 is disabled. After node 2 depletes, node 4 and node 5 broadcast DIS message to request a route maintenance. As shown in Figure 4(b), node 3 receives the DIS message from node 5 but the DIS message from node 4 cannot reach node 3 and the root. Therefore, the communication of node 5 and node 6 are still performed while node 4 is terminated. Then, it results in a network partitioning.

Figure 5 shows a diagram of data transmission of node 4 , node 5 , and node 6 when node 2 depletes. As shown in Figure 5(a), when node 2 depletes and node 3 still communicates the root node, node 4 broadcasts DIS message to neighbors. After the DIS message is transmitted, node 4 waits for an acknowledgement in a period of waiting time. After the waiting time is out, node 4 will repeat broadcasting the DIS message and waiting for acknowledgement from neighbors until its battery runs out. It can be seen that not only the data of node 4 cannot reach the root node, but also it is a waste of energy for broadcasting DIS message. On the other hand, as shown in Figures 5(b) and 5(c), the data transmission of node 5 and node 6 still reaches the root node via node 3 because node 5 can complete a route maintenance process. 


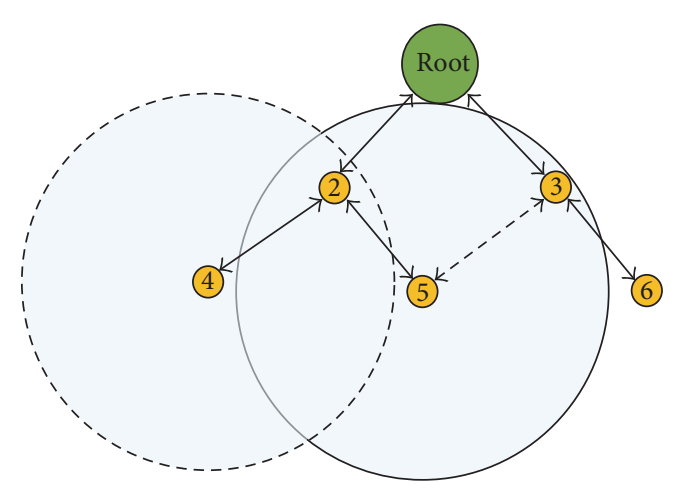

(a)

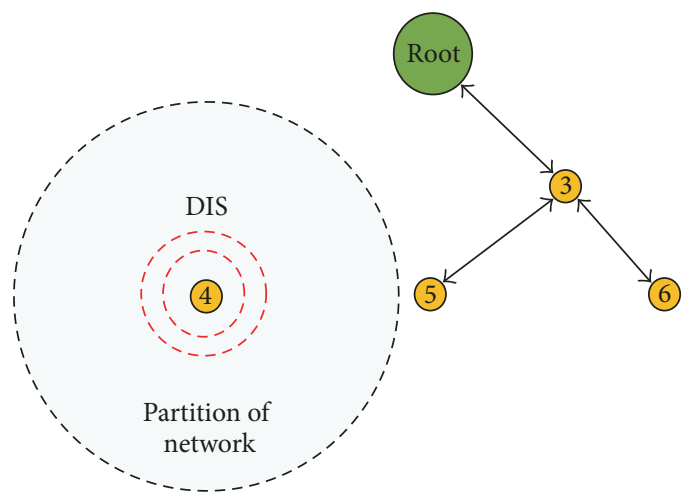

(b)

FIGURE 4: Occurrence of network partitioning, (a) network architecture of 3 ranks, and (b) network architecture of 3 ranks when node 2 is disabled.
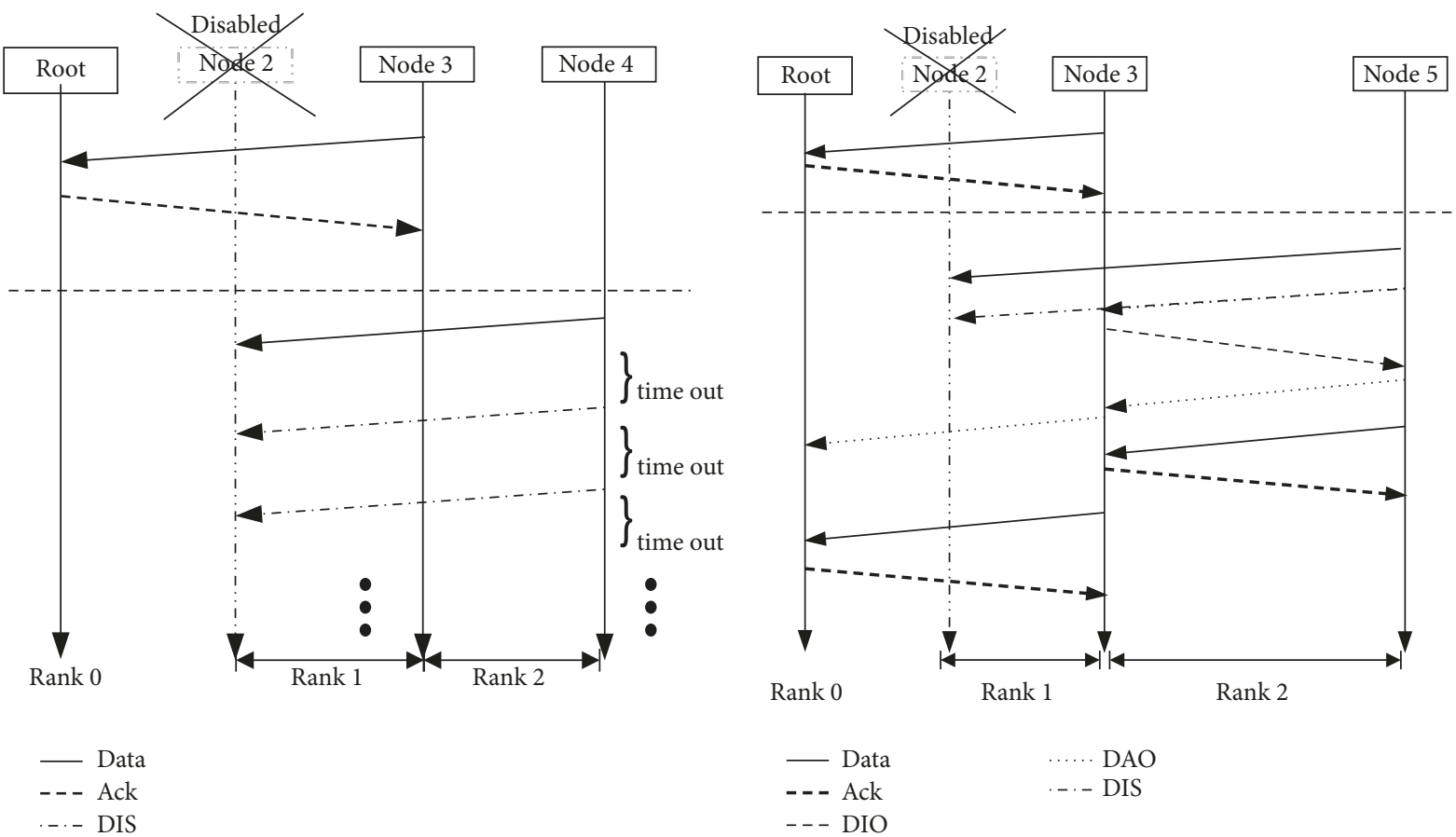

(a)

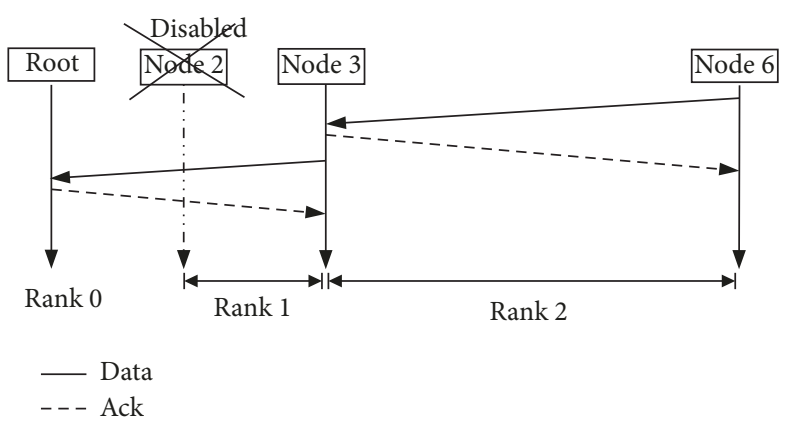

(c)

FiguRE 5: Data transmission framework of node when node 2 is disabled; (a) node 4, (b) node 5, and (c) node 6. 
Therefore, it is important to balance the workload of the nodes in the network to avoid an early depletion of node and network partitioning. Therefore, the lifetime of the lowest rank nodes (nodes 2 and 3 ) is very important for this scenario.

3.2. Transmission Reliability. In a smart grid network, even if smart meters are static, the environment such as noise, interference, and fading may affect the transmission reliability. In general, the transmission reliability is affected by two factors including node density and the quality of the transmission channel. In case of node density, when the number of nodes in the network is high, the nodes may transmit data packet at the same time and the transmission from a node may interfere with the transmission of other nodes. Then, the data packet may drop during transmission and cannot reach the parent node. Therefore, packet delivery ratio (PDR) decreases due to an increasing in the number of nodes in the network.

On the other hand, a quality of transmission channel is the main factor that affects the transmission reliability. In general, the channel quality can be determined by a ratio between received signal and noise power (SNR). In practice, the received signal power drops when the distance between nodes increases. Therefore, when a child node is far away from its parent, the SNR is poor. By this factor, the quality of received signal is worse than to be utilized and it is ignored by the parent which is referred to a decreasing in a packet delivery ratio (PDR). Then, the parent needs to request data retransmission. Since the meters have more load of data transmission, the energy consumption is higher so the battery depletes faster than expected. Therefore, the network partitioning occurs.

As mentioned earlier, we focus on the partitioned network issue; therefore, we set the network topology as a binary rooted tree architecture where the node in the same rank cannot communicate with the others due to being out of the transmission range. This kind of topology is important in practice and occurs in the rural area where each meter is far away from others; then it may communicate with its parents only. If the battery of its parent depletes, the data from the meter cannot reach the DCU. Since the meters are far away from others, the node density does not influence the transmission channel quality. Consequently, in this paper, the distance between nodes is the main factor that degrades the channel quality.

\section{Multicriteria Parent Selection Algorithm}

In this paper, we propose the multicriteria parent selection (MPS) algorithm to address two main problems-meter lifetime and stability of channel quality. Moreover, the expected transmission time (ETT) during energy usage data transmission is taken into account and used as a performance metric for selecting the immediate parent. To select the most appropriate parent, an analytic hierarchy process (AHP) is exploited by determining three critical parameters-expected transmission count (ETX), residual energy, and expected transmission time (ETT)-with assigned weight of importance. Moreover, to improve a PDR and increase an ability of workload balancing, a concept of cognitive radio (CR)

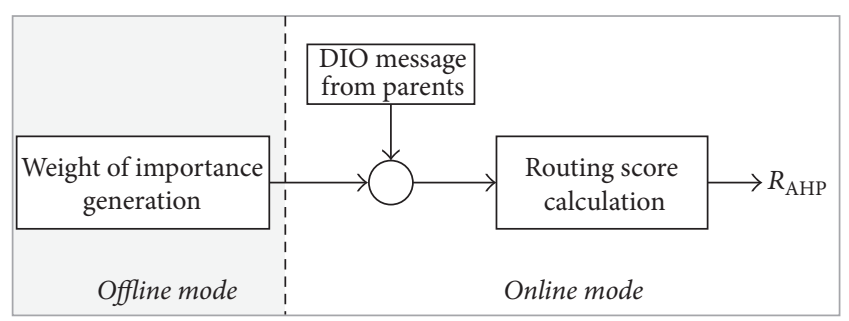

FIGURE 6: Operation of MPS mechanism.

is adopted. By utilizing concept of CR, smart meters are determined as CR enabled nodes where the meter must be implemented with CR functions. Once the bit-rate of CR channel and the transmission power are higher than Zigbee, the range of data transmission range under an acceptable PDR can be improved.

As depicted in Figure 6, the operation of MPS can be separated into an offline and online mode. In an offline mode, the weight of importance between the main criteria-ETX, residual energy, and ETT-is determined. This can be done by performing a pairwise comparison between these main criteria. Then, the weights are generated. In an online mode, MPS gathers the data-values of ETX, residual energy, and ETT-from the current and candidate parents in order to select an immediate parent. In a subcriteria phase, data from parents is compared pairwise. The results from subcriteria pairwise comparisons then performed a summation according to the predetermined weight of importance in order to compute the routing score $\left(R_{\mathrm{AHP}}\right)$ of each parent. Finally, the routing score of current and candidate parents is compared to determine the immediate parent.

Then, the framework of the multicriteria parent selection (MPS) algorithm is categorized into two sections-parent selection during route construction and parent selection during energy usage data transmission.

4.1. Parent Selection during Route Construction. As stated by RPL provision mechanism, a control message is broadcast over network continually in order to form a network topology dynamically. As described in the previous section, once a network topology is formed, both parent node and candidate node send a DIO message to higher rank nodes where the higher rank nodes are determined as children. After a child receives a DIO message, it will determine which node is appropriate to be its parent. As mentioned earlier, to select the parent, route performance-ETX_cannot be merely determined. Therefore, other metrics-residual energy and ETT-need to be considered.

In this subsection, a routing selection using AHP algorithm is described. AHP is a powerful tool that combines several indicators performance into a single key performance indicator (or performance metrics) [32, 33]. Therefore, different weight of importance between performance indicators should be assigned to those performance indicators. By determining the weight using AHP, a pair comparison between indicators is determined as the weight. Therefore, an inconsistency of parent selecting can be addressed. To 


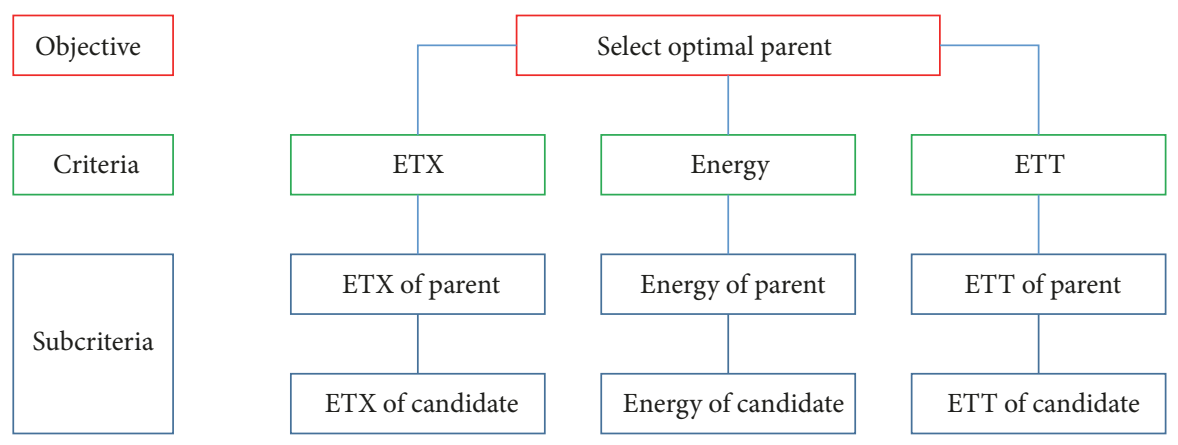

FIGURE 7: Structure of objective, criteria, and subcriteria.

determine the most appropriate parent while maintaining overall performance, ETX, residual energy, and ETT are determined as the parent selection criteria of AHP.

Then, the routing score of each parent can be determined as the following procedures:

(1) Define the objective. In this paper, the objective is to determine the most appropriate parent when ETX, residual energy and ETT are determined as the criteria. The subcriteria are the real value of ETX in each route, residual energy of parent, and the ETT of the route.

(2) Group the criteria, subcriteria, and alternatives. As described in the previous procedure, the structure of objective, criteria, and subcriteria is shown in Figure 7.

(3) Make a pairwise comparison of elements in each group.

(3.1) Construct $n \times n$ of pairwise comparison matrix (A), where $n$ is the number criteria, by comparing each criteria with respect to others. Then, the pairwise comparison matrix $(\mathbf{A})$ is shown in Figure 8 and can expressed as

$$
\mathbf{A}=\left[\begin{array}{ccc}
1 & a_{12} & a_{13} \\
a_{21} & 1 & a_{23} \\
a_{31} & a_{32} & 1
\end{array}\right],
$$

where $a_{12}$ represents the weight of importance between ETX and remaining energy, $a_{13}$ represents the weight of importance between ETX and ETT, and $a_{23}$ represents the weight of importance between remaining energy and ETT.

It should be noted that $a_{j i}$ is equal to $1 / a_{i j}$ when $i$ and $j$ are $1,2, \ldots, n$. To compare the weight of important between criteria, the scale of range 9 to $1 / 9$ is determined. For example, if $a_{12}$ is 5 , ETX is 5 times more important than energy. On the other hand, if $a_{12}$ is $1 / 5$, ETX is 5 times less important than energy.

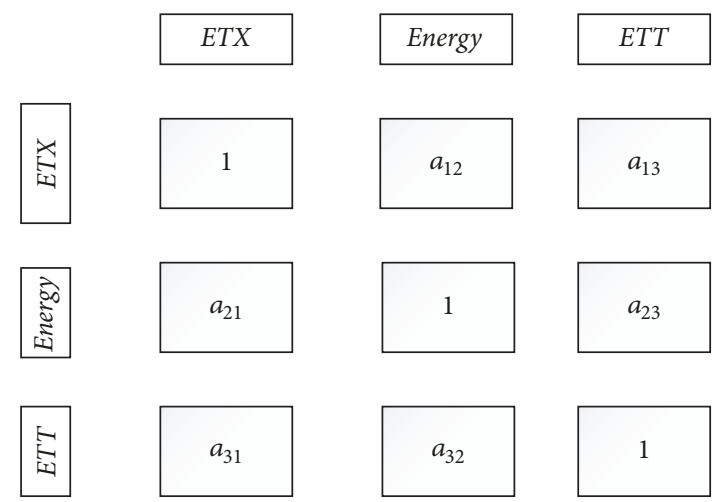

FIgURe 8: Pairwise comparison matrix (A) of main criteria.

(3.2) Compute the normalize eigenvector. Then, the weight of importance of each criteria $\left(\mathbf{c}=\left[c_{1}, c_{2}, \ldots, c_{n}\right]\right)$ is obtained.

(3.3) Do procedures (3.1) and (3.2) for subcriteria of each criteria. It should be noted that the weight of importance of subcriteria can be determined by the ratio of real values of compared elements.

(4) Calculate the weight of subcriteria according to the main criteria.

(5) Evaluate the alternatives according to the weight by summarizing all of calculated weights for each element.

4.2. Parent Selection during Energy Usage Data Transmission. In an AMI network, the node (meter) has to send the information about energy usage every 15 minutes to the DCU for real-time processing of smart grid technology. In this subsection, we propose a CR hopping algorithm where the nodes determine the worthiness of exploiting available channel of CR network when ETX, ETT, and residual energy are determined. As illustrated in Algorithm 1, the parameters - residual energy, ETX, and ETT—of immediate parent and candidate parent are used as the inputs of the algorithm. It should be noted that subscripts "im" and " $c$ " are an immediate parent and candidate parent, respectively. $P_{i}$ denotes the serviced parent. $\mathrm{CH}_{i}$ denotes the transmission 


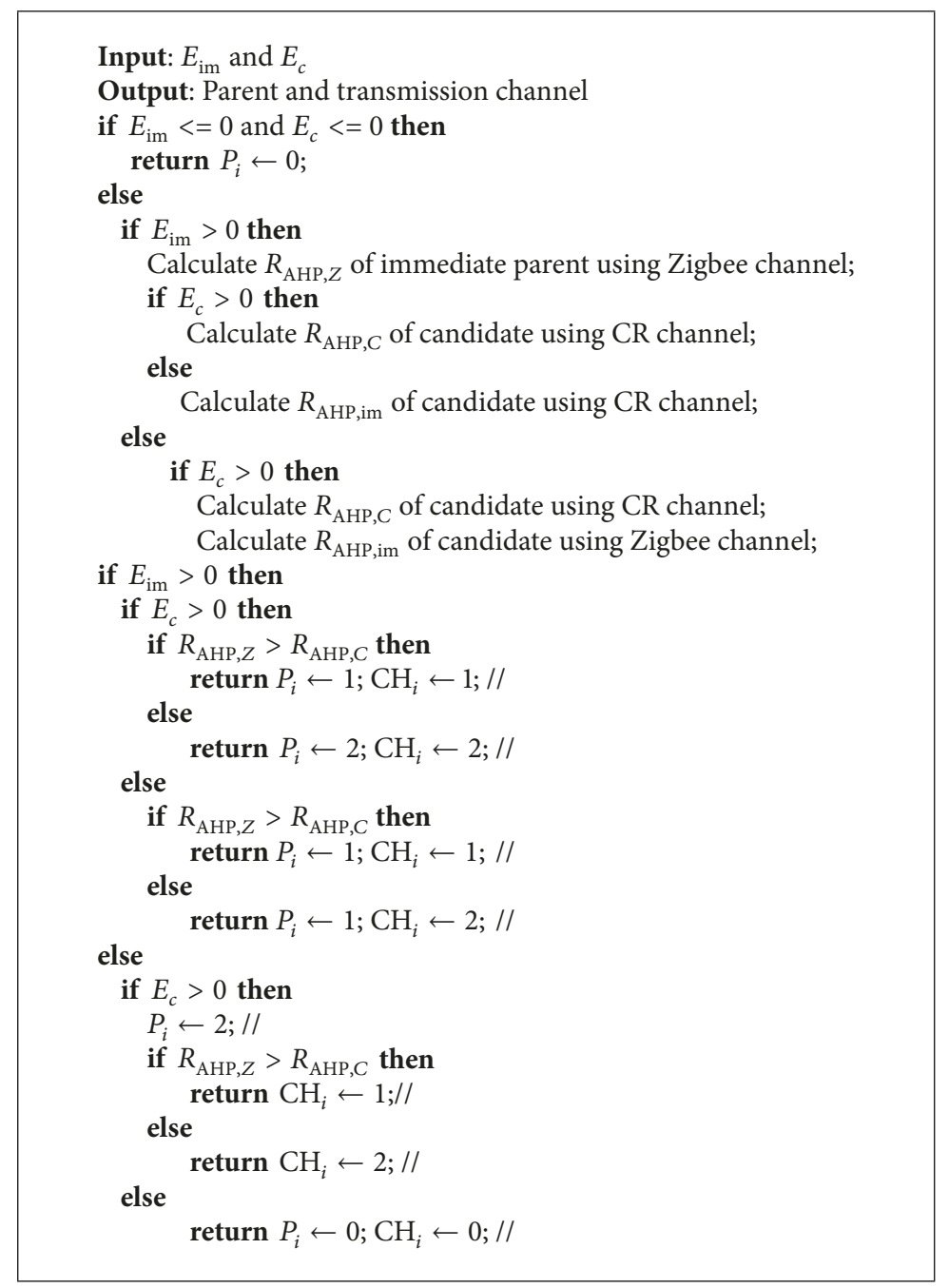

Algorithm 1: CR Hopping during importance data transmission.

protocol where Zigbee and CR are determined. $R_{\mathrm{AHP}}$ is a calculated routing score of parent and candidate parent.

First, residual energy of immediate and candidate parent is determined. If the immediate and candidate parent deplete, the algorithm states that there is no enabled parent. Otherwise, the residual energy of immediate and candidate parent is compared to assign their transmission technology. If both immediate and candidate parent are enabled, the Zigbee and $\mathrm{CR}$ are assigned to immediate and candidate parent, respectively. If energy of either immediate or candidate parent is enabled, Zigbee and CR are assigned to the enabled parent, to calculate the routing score. Therefore, the routing score of enabled parent with Zigbee and routing score of enabled parent with CR technology is determined by $R_{\mathrm{AHP}, Z}$ and $R_{\mathrm{AHP}, \mathrm{C}}$, respectively. Second, after transmission technology is assigned to both immediate and candidate parent, the routing score is calculated by using AHP algorithm as described in the previous subsection. Then, the routing scores are compared. The output of the algorithm declares two parameters including selected parent and the transmission technology where the interpretation of subscripted number of (i) is shown in Table 1.
In an information interaction overhead, in RPL protocol, the DIO message for route construction and maintenance is exchanged between nodes periodically. Our mechanism also follows this protocol; it does not incur any extra overhead. To select a parent in the route maintenance, the main computation of our mechanism is on the pairwise comparison of subcriteria, where an eigendecomposition theory is utilized. The process is bound at $O\left(n^{3}\right)$ where $n$ is number of candidate parents.

\section{Simulation Results}

In this section, the performance of the multicriteria parent selection (MPS) algorithm is compared to two early parent selecting functions-traditional RPL and energy-efficient oriented algorithm (EERA) [20]. As mentioned earlier, the main objective of RPL protocol on smart grid technology is to distribute an information over a network topology while maintaining a system reliability using low-power devices and prolonging the meter lifetime. Therefore, the two vital performance metrics that are used to evaluate the network 
TABLE 1: Interpretation of subscripted number.

\begin{tabular}{lccc}
\hline Subscripted number $(i)$ & 0 & 1 & 2 \\
\hline $\mathrm{CH}_{i}$ & No enabled technology & Zigbee & $\mathrm{CR}$ \\
$P_{i}$ & No enabled parent & Immediate parent & Candidate parent \\
\hline
\end{tabular}

TABLE 2: Simulated parameters.

\begin{tabular}{lc}
\hline Parameters & Value \\
\hline Tx power & $66.16 \mathrm{~mW}$ \\
Rx power & $70.695 \mathrm{~mW}$ \\
Tx power in CR mode & $198.51 \mathrm{~mW}$ \\
Idle power & $900 \mathrm{nW}$ \\
Sleep power & $400 \mathrm{nW}$ \\
Spectrum sensing power & $65.83 \mathrm{~mW}$ \\
DIO interval before data transmitting & 5 seconds \\
Data transmitting interval & 5 mins \\
Data packet & 127 bytes \\
DIO message size & 64 bytes \\
DAO message size & 46 bytes \\
DIS message size & 2 bytes \\
Transmission time of data packet & $192 \mathrm{us}$ \\
Transition time from sleep to active mode & $970 \mathrm{us}$ \\
\hline
\end{tabular}

performance are packet delivery ratio (PDR) and the meter lifetime of the network.

5.1. Simulation Setup. In this paper, the two communication schemes-route construction and energy usage data communication-are determined. It should be noted that the notification alert and the performance of ETT will be considered in the future work. All simulation runs on MATLAB where the simulation time is 3600 minutes. Parameters which are set in the simulation are shown in Table 2 .

As shown in Figure 1, the network topology is set as a binary rooted tree architecture and defined that the quality of left transmission channel is better than the right one defined by the distance between two meters. For example, for 3 ranks, the distance from node 5 to node 2 is less than node 5 to node 3 as depicted in Figure 9. In the scenario, the node in the same rank cannot communicate with the others due to the transmission range. Then, the consumption energy can be calculated by using (3). Moreover, we also investigate the transmission quality due to an increasing of transmission range. Then, the distance between nodes ranged between 10 and 100 meters.

Since one ancestor depletes, it means that the data transmission of children is terminated. Therefore, the meter lifetime is measured only when the data transmission can reach to the DCU. To evaluate the meter lifetime, the maximum, average, and minimum meter lifetime as a function of rank numbers are illustrated where the maximum, average, and minimum lifetime are obtained from all meters. The meter lifetime is determined by the time that the communication of a meter cannot reach the DCU.

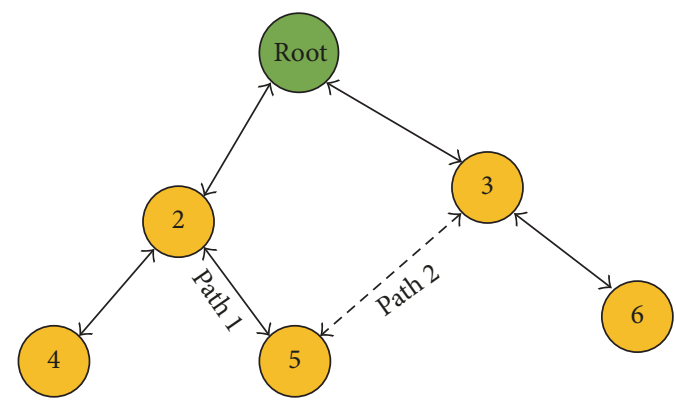

FIGURE 9: 3-rank network topology.

Workload balancing ability is considered through a relative balancing factor (RBF) which is given as

$$
\begin{aligned}
\mathrm{BF}_{i} & =\mid \text { Lifetime }_{\text {max }}-\text { Lifetime }_{\text {min }} \mid \\
\mathrm{RBF} & =\frac{\left|\mathrm{BF}_{\mathrm{RPL}}-\mathrm{BF}_{i}\right|}{\mathrm{BF}_{\mathrm{RPL}}},
\end{aligned}
$$

where $\mathrm{BF}$ is a balancing factor in each parent selection function, Lifetime $e_{\max }$ is the maximum lifetime of node in each rank, Lifetime min $_{\text {is }}$ is the minimum lifetime of node in each rank, and $i$ is the EERA or MPS.

To compare the improvement of partitioned network avoiding, the minimum meter lifetime of later parent selecting function is compared with respect to the minimum meter lifetime of traditional RPL. Then, the percentage of improvement (PM) can be expressed as

$$
\mathrm{PM}=\left(\frac{\mid \text { Lifetime }_{\text {min,RPL }}-\text { Lifetime }_{\text {min }, i} \mid}{\text { Lifetime }_{\text {min, RPL }}}\right) \times 100,
$$

where Lifetime $e_{\min }$ is the minimum lifetime of node in each network and $i$ is the EERA or MPS.

In order to compute the weights, every two factors performed pairwise comparison where the important weight between two factors need to be assigned. In this paper, we investigate the appropriate value of important weights and those values are set as follows: the importance between ETX and residual energy is equal and the ETX and residual energy are 2 times more important than ETT. Then, the pairwise comparison matrix $(\mathbf{A})$ is given by

$$
\mathbf{A}=\left[\begin{array}{lll}
1 & 1 & 2 \\
1 & 1 & 2 \\
\frac{1}{2} & \frac{1}{2} & 1
\end{array}\right] .
$$




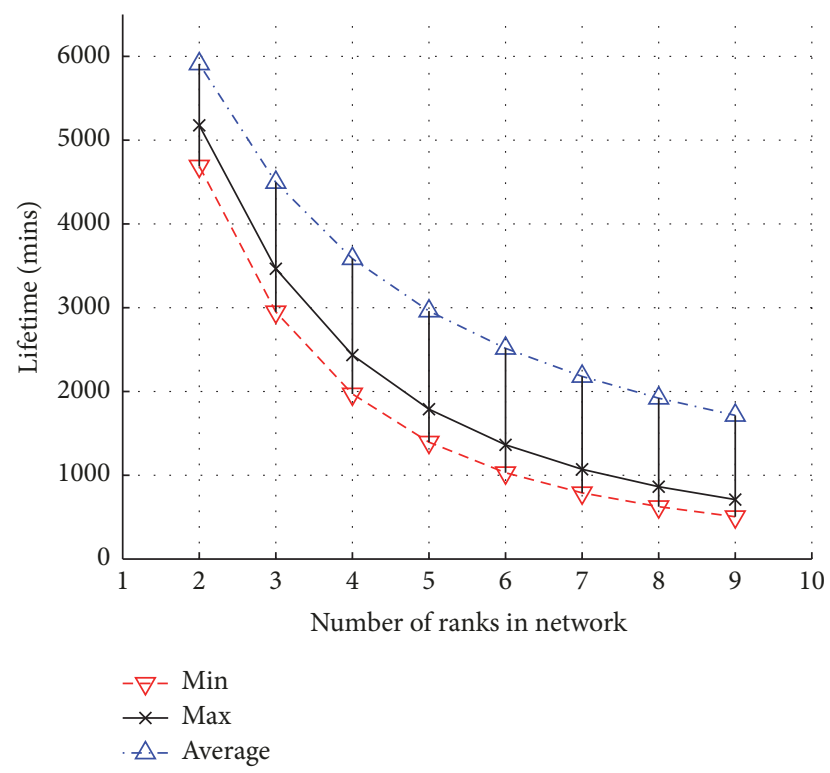

FIGURE 10: Node operation time of traditional RPL mechanism.

5.2. Simulation Results. In this subsection, we first evaluate the performance of three parent selection functionstraditional RPL (RPL), EERA, and MPS-under different number of ranks.

First, the meter lifetime of each parent selection function is illustrated. As shown in Figure 10, by using traditional RPL (RPL), the maximum meter lifetime is much different from the minimum. Because the child meters keep forwarding the data to the parent who is nearer to it, for example, node 5 forwards the data to node 2 until the battery of node 2 depletes. After node 2 depletes, the data from node 4 cannot be transmitted to the DCU. Then, node 4 is partitioned from the network. On the other hand, before node 2 depletes, node 3 has only one child, node 6 . After node 2 depletes, node 3 is determined as a relay of nodes 5 and 6 . Therefore, the average meter lifetime of traditional RPL decreases when the number of ranks increases. As shown in Figure 11, since EERA selects the immediate parent by considering residual energy of both candidate parents and ETX. Therefore, the workload of parents is balanced since the maximum meter lifetime is near to the minimum. Moreover, it can be seen that the average meter lifetime increases as compared to the traditional RPL.

As shown in Figure 12, for MPS algorithm, it can be seen that the maximum meter lifetime of MPS is nearly the same as the minimum which means that MPS balance the meter workload in the network efficiently. This is because MPS considers the three performance metrics-ETX, residual energy, and ETT - as the multiobjective and performing a pairwise comparison between them. Then, the appropriate parent is analyzed comprehensively. During data transmission, even if the CR mode is activated and it consumes more energy than Zigbee, MPS can still balance the meter workload in network.

As shown in Figure 13, the improvement of balancing ability of MPS and EERA is compared to traditional RPL

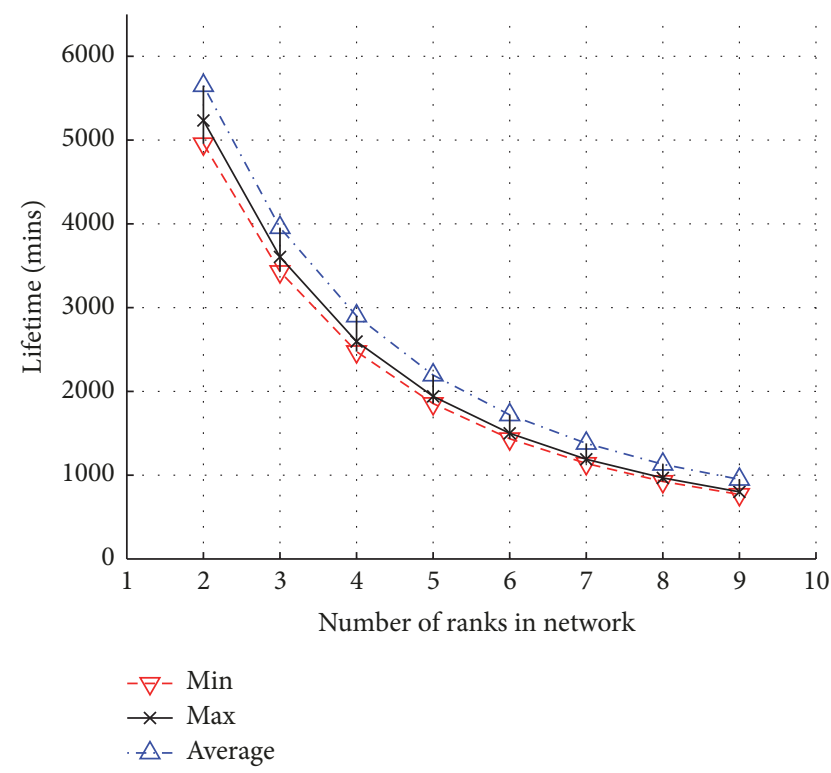

FIgURE 11: Node operation time of EERA.

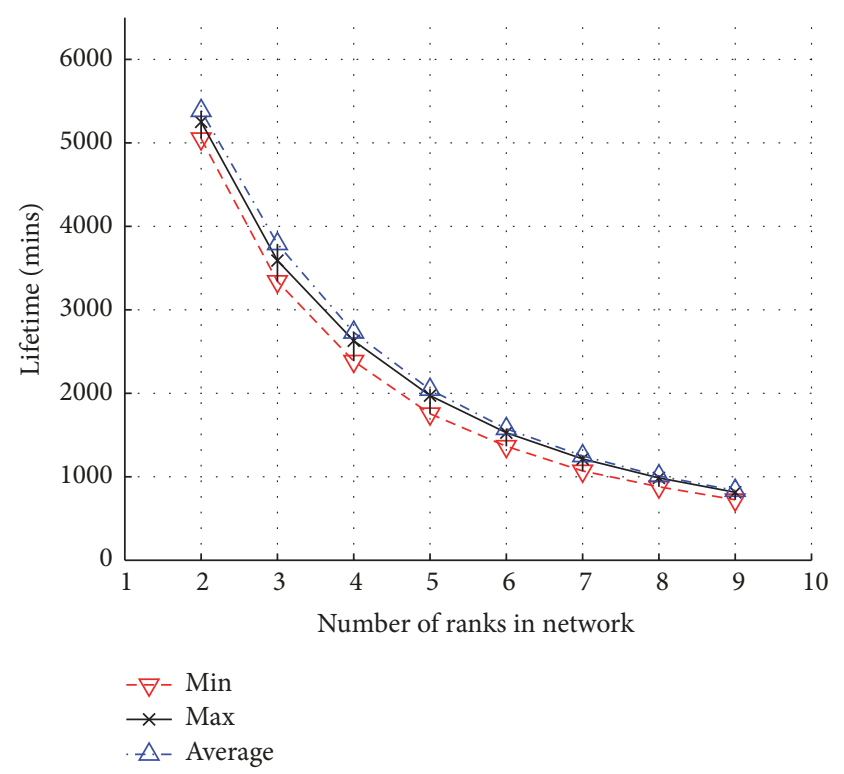

FIGURE 12: Node operation time of MPS.

through a relative balancing factor which can be expressed in (6). As the number of ranks increases, the number of children of the highest ancestors (nodes 2 and 3 ) increases. Then, the highest ancestors consumes more energy, once they have to pass on the children data to the root node. Since traditional RPL ignores the residual energy of the lower rank nodes to determine the immediate parent, the workload of parent nodes is not balanced. Therefore, when MPS and EERA take residual energy into account, they give better balancing ability than traditional RPL. As compared to EERA, MPS gives higher improvement of balancing ability for all ranks because MPS improves the balancing ability by considering 


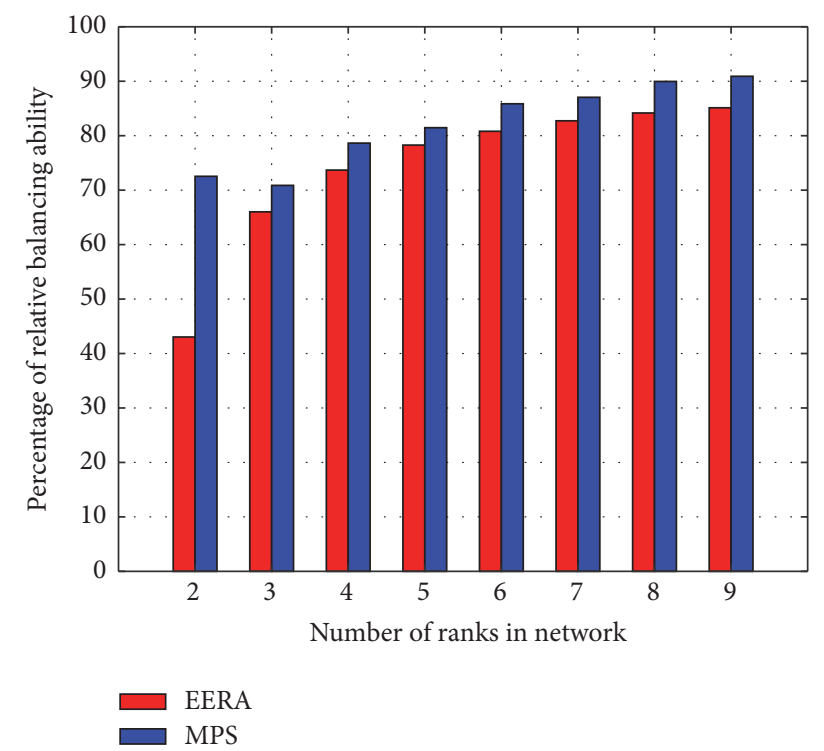

FIGURE 13: A balancing factors of MPS as compared to traditional RPL and EERA.

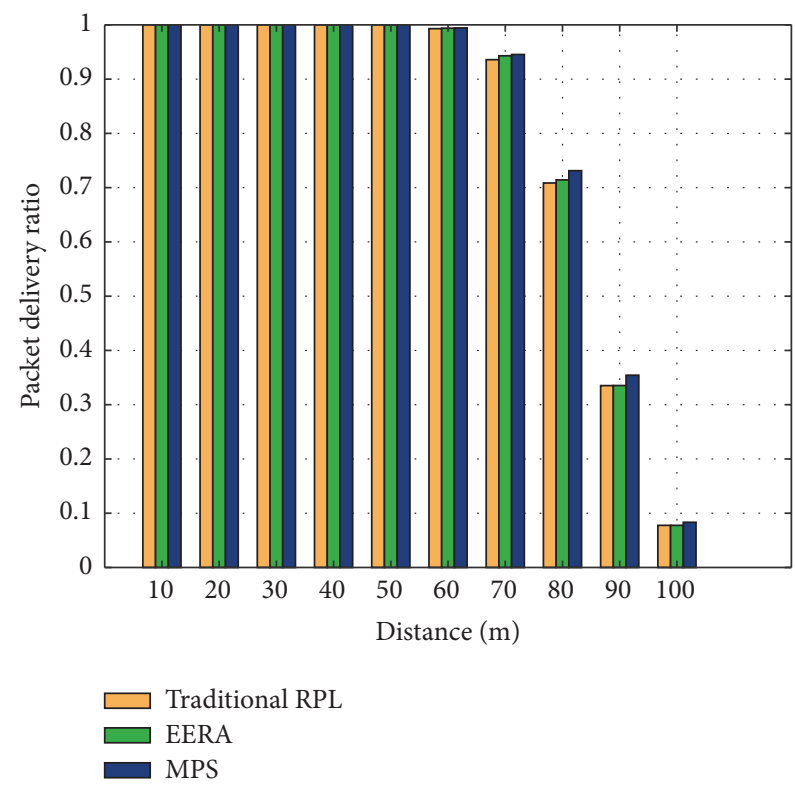

FIgURE 14: Average PDR of MPS as compared to RPL and EERA during route construction.

the routing factors more comprehensively and adopting CR channel.

Second, we evaluate the transmission reliability by comparing the average PDR as a function of distances. As shown in Figure 14, an average PDR decreases due to an increase in distance between nodes. It should be noted that, during route construction and maintenance, MPS does not activate CR mode for battery saving purpose. By using analyzing weights using AHP, not only the workload of parents is balanced, but also MPS gives the highest averaged PDR for all distances.

Third, we evaluate the transmission reliability during energy usage data transmission by comparing the average

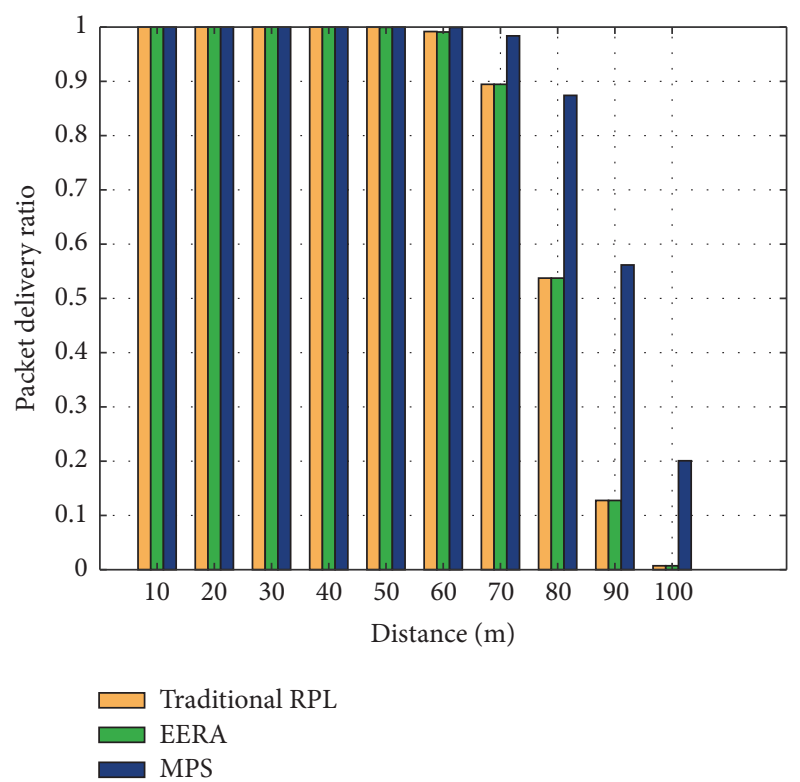

FIgURE 15: Average PDR of MPS as compared to RPL and EERA during energy usage data transmission.

TABLE 3: Percentage of network partitioning avoidance.

\begin{tabular}{lcc}
\hline Rank & EERA & MPS \\
\hline 2 & $7.675 \%$ & $5.65 \%$ \\
3 & $13.22 \%$ & $16.27 \%$ \\
4 & $21.06 \%$ & $25.63 \%$ \\
5 & $25.80 \%$ & $32.97 \%$ \\
6 & $32.52 \%$ & $39.32 \%$ \\
7 & $35.44 \%$ & $44.30 \%$ \\
8 & $40.80 \%$ & $48.00 \%$ \\
9 & $43.56 \%$ & $52.47 \%$ \\
\hline
\end{tabular}

PDR as a function of distances. As a result, the size of packet affects the PDR. As compared to route construction period, the PDR during data transmission is lower than route construction at the same distance. As shown in Figure 15, by adopting CR technology, the PDR of MPS is much greater than traditional RPL and EERA. When MPS activates the CR mode, it transmits the data on the different spectrum frequency of Zigbee. Therefore, it has lower interference than using Zigbee channel. Moreover, based on different protocol, the transmission power on the CR mode is greater than Zigbee mode. Then, PDR can be improved.

Moreover, we also evaluate the ability of communication network partitioning avoidance as compared to the traditional RPL. As depicted in Table 3, by taking a residual energy into the parent selection account, MPS and EERA improve the performance of partitioned network avoiding of traditional RPL. Since MPS determines the weights using pair comparison between two performance metrics, MPS can avoid partitioned network better than EERA. 


\section{Conclusion}

In this paper, a novel parent selection function-multicriteria parent selection (MPS) algorithm-is explored to address the issue of network partitioning and improves the transmission reliability of early parent selection functions. The three routing factors are used as the parent selection constraints; therefore, MPS can balance the workload of meters in AMI network and avoids the network partitioning efficiently. By considering the three routing factors as the parent selection constraints, the routing factors are analyzed more comprehensively than early parent selection algorithms. Moreover, we investigate the appropriate values for pairwise comparing between factors to generate the appropriate weights and those values are reported. Once the workload is balanced, the lifetime of meters in network is nearly the same and can be predicted easily. Then, it is fully facilitated to the maintenance team for planning the network maintenance time. Moreover, by adopting cognitive radio technology, the data is transmitted in an unused channel opportunistically where the channel quality is better than Zigbee. Therefore, MPS improves the transmission reliability and also reduces the number of data retransmissions.

\section{Conflicts of Interest}

The authors declare that they have no conflicts of interest.

\section{References}

[1] V. C. Gungor, D. Sahin, T. Kocak et al., "A survey on smart grid potential applications and communication requirements," IEEE Transactions on Industrial Informatics, vol. 9, no. 1, pp. 28-42, 2013.

[2] V. C. Güngör, D. Sahin, T. Kocak et al., "Smart grid technologies: communication technologies and standards," IEEE Transactions on Industrial Informatics, vol. 7, no. 4, pp. 529-539, 2011.

[3] U. Ozgur, S. Tonyali, K. Akkaya, and F. Senel, "Comparative evaluation of smart grid AMI networks: performance under privacy," in Proceedings of the 2016 IEEE Symposium on Computers and Communication, ISCC, pp. 1134-1136, Italy, July 2016.

[4] L. Hernandez, C. Baladron, J. M. Aguiar et al., "A survey on electric power demand forecasting: Future trends in smart grids, microgrids and smart buildings," IEEE Communications Surveys \& Tutorials, vol. 16, no. 3, pp. 1460-1495, 2014.

[5] P. Sethi and S. R. Sarangi, "Internet of things: architectures, protocols, and applications," Journal of Electrical and Computer Engineering, vol. 2017, Article ID 9324035, pp. 1-25, 2017.

[6] D. F. Ramírez, S. Céspedes, C. Becerra, and C. Lazo, "Performance evaluation of future AMI applications in Smart Grid Neighborhood Area Networks," in Proceedings of the 2015 IEEE Colombian Conference on Communications and Computing, COLCOM, Colombia, May 2015.

[7] S. Nimbargi, S. Mhaisne, S. Nangare, and M. Sinha, "Review on AMI technology for Smart Meter," in Communication and Computer Technology (ICAECCT, pp. 21-27, Pune, 2016 IEEE International Conference on Advances in Electronics.

[8] D. Wang, Z. Tao, J. Zhang, and A. A. Abouzeid, "RPL based routing for advanced metering infrastructure in smart grid," in Proceedings of the 2010 IEEE International Conference on Communications Workshops, ICC, South Africa, May 2010.
[9] T. Winter, P. Thubert, A. Brandt et al., "RPL: IPv6 routing protocol for low-power and lossy networks," J. Chem. Inf. Model, vol. 53, no. 9, pp. 1689-1699, 2012, RFC6550, s.l.: IETF, http://tools.ietf.org/html/rfc6.

[10] I. Ishaq, D. Carels, G. Teklemariam et al., "IETF standardization in the field of the internet of things (IoT): a survey," Journal of Sensor and Actuator Networks, vol. 2, no. 2, pp. 235-287, 2013.

[11] A. Kheaksong, K. Srisomboon, and W. Lee, "A comparative study of various routing protocols for smart grid communication," ECTI E-Magazine, vol. 10, no. 1, 2016.

[12] H. Tian, Z. Qian, X. Wang, and X. Liang, "QoI-Aware DODAG Construction in RPL-Based Event Detection Wireless Sensor Networks," Journal of Sensors, vol. 2017, Article ID 1603713, 2017.

[13] P. Thubert, “Objective Function Zero for RPL," RFC 6552, 2012.

[14] A. S. Sadiq, T. Z. Almohammad, R. A. B. M. Khadri, A. A. Ahmed, and J. Lloret, "An Energy-Efficient Cross-Layer approach for cloud wireless green communications," in Proceedings of the 2nd International Conference on Fog and Mobile Edge Computing, FMEC, pp. 230-234, Spain, May 2017.

[15] W. Zhang, G. Han, Y. Feng, and J. Lloret, "IRPL: An energy efficient routing protocol for wireless sensor networks," Journal of Systems Architecture, vol. 75, pp. 35-49, 2017.

[16] A.-L. Kampen, K. Øvsthus, and Ø. Kure, "Energy balancing algorithms in wireless sensor networks," in Proceedings of the Federated Conference on Computer Science and Information Systems, FedCSIS, pp. 1223-1231, Poland, September 2015.

[17] O. Gnawali and P. Levis, "The Minimum Rank with Hysteresis Objective Function," Internet Requests for Comments RFC6719, 2012.

[18] E. Ancillotti, R. Bruno, and M. Conti, "Reliable data delivery with the IETF routing protocol for low-power and lossy networks," IEEE Transactions on Industrial Informatics, vol. 10, no. 3, pp. 1864-1877, 2014.

[19] N. Pradeska, Widyawan, W. Najib, and S. S. Kusumawardani, "Performance analysis of objective function MRHOF and OF0 in routing protocol RPL IPV6 over low power wireless personal area networks (6LoWPAN)," in Proceedings of the 8th International Conference on Information Technology and Electrical Engineering, ICITEE, Indonesia, October 2016.

[20] L.-H. Chang, T.-H. Lee, S.-J. Chen, and C.-Y. Liao, "Energyefficient oriented routing algorithm in wireless sensor networks," in Proceedings of the IEEE International Conference on Systems, Man, and Cybernetics, SMC, pp. 3813-3818, October 2013.

[21] P. M. Esposito, M. E. M. Campista, I. M. Moraes, L. H. M. K. Costa, O. C. M. B. Duarte, and M. G. Rubinstein, "Implementing the expected transmission time metric for OLSR wireless mesh networks," in Proceedings of the 1st IFIP Wireless Days (WD '08), pp. 1-5, IEEE, Dubai, UAE, November 2008.

[22] J. Mitola, "Cognitive radio architecture evolution," Proceedings of the IEEE, vol. 97, no. 4, pp. 626-641, 2009.

[23] S. Haykin, "Cognitive radio: brain-empowered wireless communications," IEEE Journal on Selected Areas in Communications, vol. 23, no. 2, pp. 201-220, 2005.

[24] S. Bayhan, S. Eryigit, F. Alagöz, and T. Tugcu, "Low complexity uplink schedulers for energy-efficient cognitive radio networks," IEEE Wireless Communications Letters, vol. 2, no. 3, pp. 363-366, 2013.

[25] C. Cordeiro, K. Challapali, D. Birru, and N. Sai Shankar, "IEEE 802.22: An introduction to the first wireless standard based on cognitive radios," Journal of Communications, vol. 1, no. 1, pp. 38-47, 2006. 
[26] N. R. Smith and K. Humood, An efficient scheme in IEEE 802.22. WRAN for Real time and Non-Real time. Traffic delay, Blekinge Institute of Technology, 2013.

[27] A. H. Process, “The Analytic Hierarchy Process," Computing, 1980.

[28] R. W. Saaty, "The analytic hierarchy process-what it is and how it is used," Applied Mathematical Modelling: Simulation and Computation for Engineering and Environmental Systems, vol. 9, no. 3-5, pp. 161-176, 1987.

[29] "Analytic hierarchy process (AHP) tutorial," [Online]. Available: http://people.revoledu.com/kardi/tutorial/AHP/AHP.htm.

[30] W. Lee, K. Srisomboon, and A. Prayote, "Fast spectrum sensing with coordinate system in cognitive radio networks," ETRI Journal, vol. 37, no. 3, pp. 491-501, 2015.

[31] P. Levis, T. Clausen, J. Hui, O. Gnawali, and J. Ko, “The Trickle Algorithm," Tech. Rep. RFC6206, 2011.

[32] S. Ajami and S. Ketabi, "Performance evaluation of medical records departments by analytical hierarchy process (AHP) approach in the selected hospitals in Isfahan: Medical Records Dep. \& AHP," Journal of Medical Systems, vol. 36, no. 3, pp. 11651171, 2012.

[33] Y. Min, L. T. Yang, F. Wang, and W. Wang, "Dynamic Sleeping Algorithm based on AHP for wireless sensor networks," in Proceedings of the 2nd International Conference on Future Generation Communication and Networking, FGCN, pp. 387392, China, December 2008. 


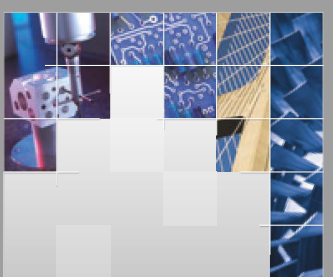

\section{Enfincering}
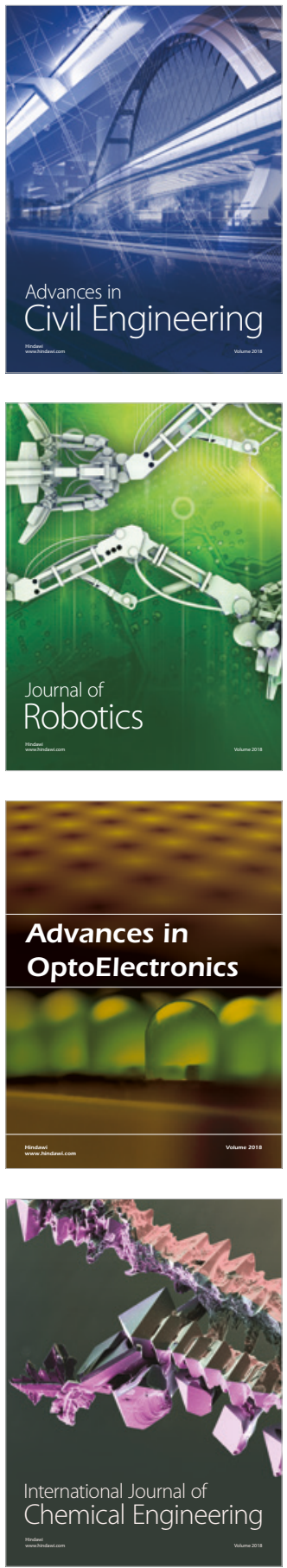

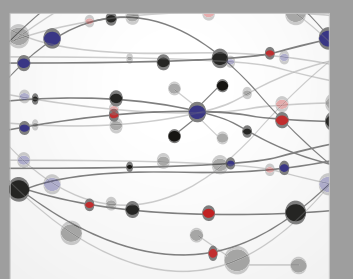

\section{Rotating \\ Machinery}

The Scientific World Journal

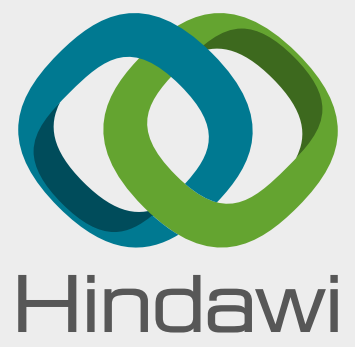

Submit your manuscripts at

www.hindawi.com
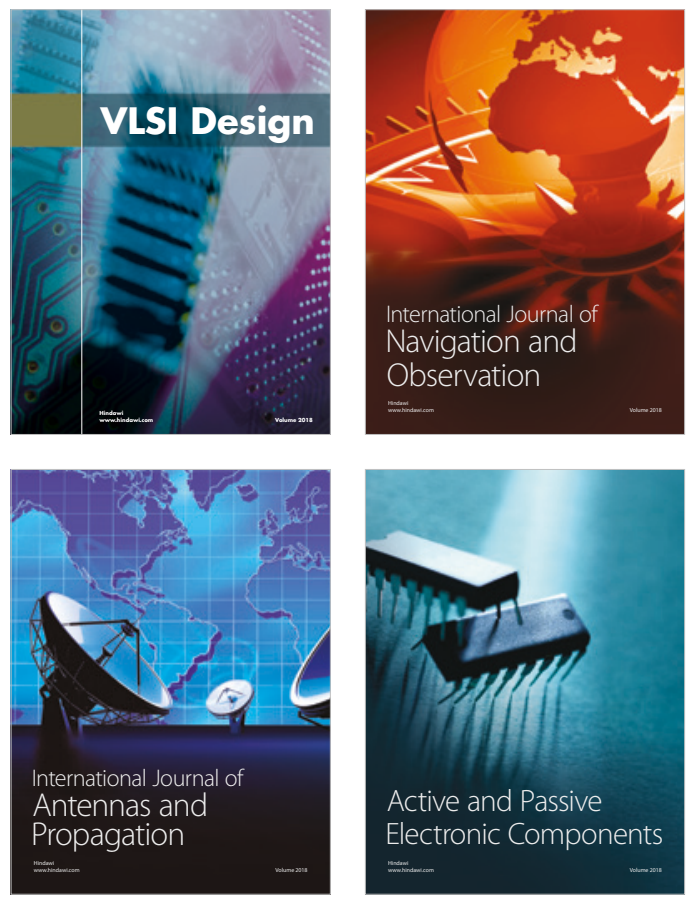
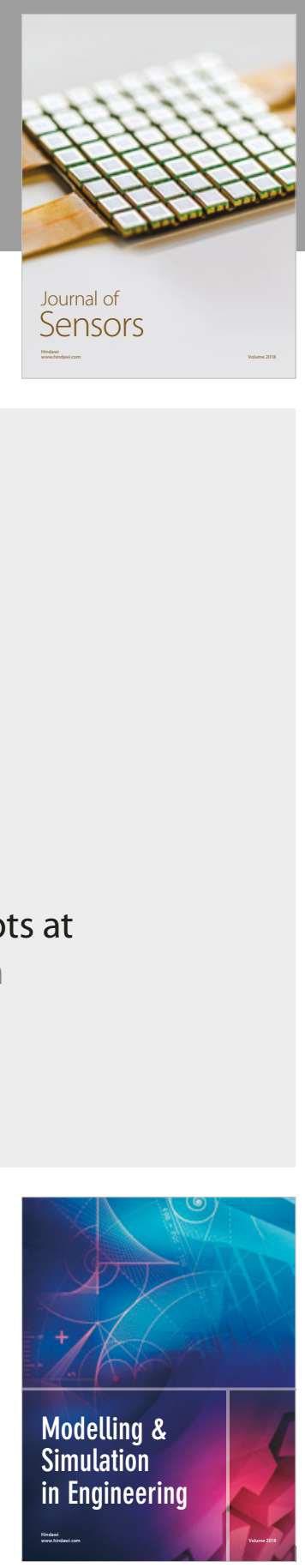

\section{Advances \\ Multimedia}
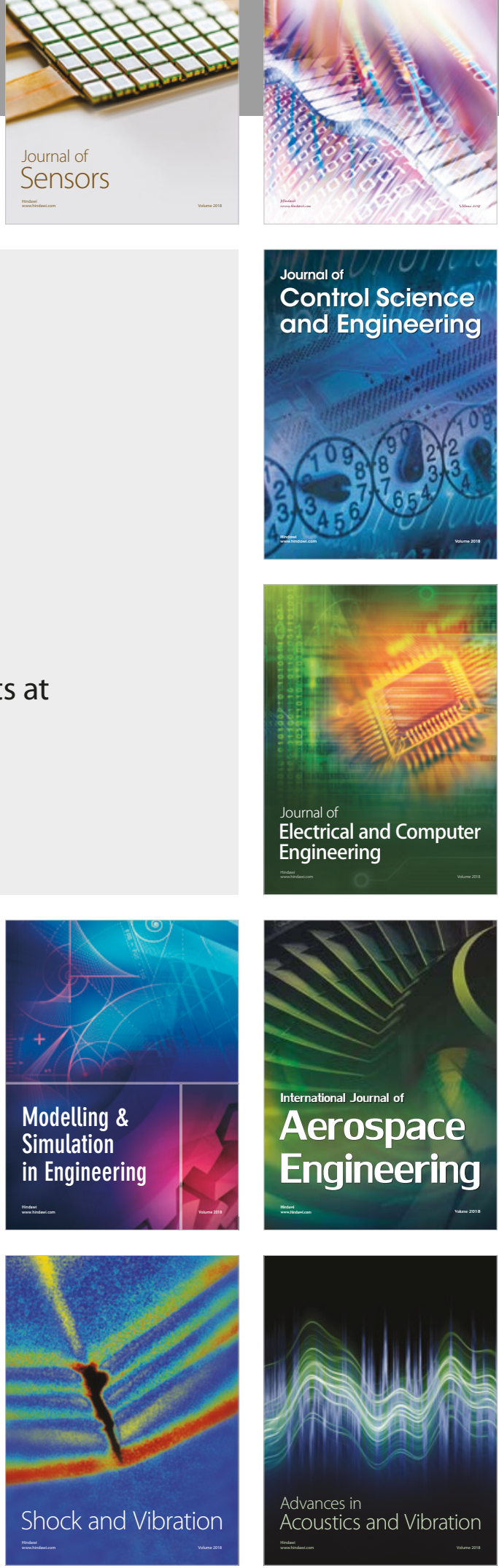